\title{
Concepts of carrying capacity and substitution ratios: a
} systems viewpoint

\author{
DAVID L. SCARNECCHIA
}

\section{Abstract}

Usefulness of the concepts of carrying capacity and species substitution ratios in natural resource management has been limited by single objectives and single management options implicit in their definitions. When applied to livestock, they have been further limited by poor conceptualization of the animal-unit concept often used to quantify them. A systematic approach to the animal-unit concept logically leads to concepts of livestock carrying capacity and livestock substitution ratios compatible with the multiple objectives and multiple management options characteristic of range livestock systems. This paper develops simplified multi-objective concepts of carrying capacity and substitution ratios, and discusses their interpretation and application in agroecological systems. Also discussed is the applicability of these concepts to describe components other than livestock in managed and unmanaged systems. Some thoughts are presented on abstraction and simplification of concepts.

Key Words: animal-unit, animal-unit-month, grazing capacity, optimum stocking rate, stocking level, terminology

The concept of carrying capacity has been widely and diversely used in natural resource management. In range livestock systems, it and the sometimes synonymous grazing capacity have often referred to the number of animals which an area of land can support without degradation of plant or soil resources. Working from this general definition, attempts have been made to develop substitution ratios of different species of livestock, express them in common animal-units $(\mathrm{AU})$, and then match the animal-units stocked over some time (the stocking level) (AUM/ha) to an estimated carrying capacity. The usefulness, if not the use, of the carrying capacity concept applied to livestock has been limited by (1) a single or dual objective approach to its definition (i.e., maintain livestock and/or maintain plant and soil resources), (2) unclear concepts of the animal-unit and animal-unit-equivalent often used in calculating stocking levels and expressing carrying capacities, and (3) poor conceptualization of species substitution ratios and their relationships to both animal-unit equivalents and carrying capacity.

Recent papers have outlined systematic concepts of the animalunit and animal-unit equivalent (Scarnecchia 1985a, Scarnecchia 1986, Scarnecchia and Gaskins 1987) which logically lead to multiobjective concepts of substitution ratios and carrying capacity. These concepts are rigorous and simplified, and meet the particular needs of range livestock systems and the general requirements of ecology. This paper presents redesigned multi-objective concepts of carrying capacity and substitution ratios, and discusses their interpretations and applications in range livestock and other systems.

\footnotetext{
Author is associate professor, Department of Natural Resource Sciences, Washington State University, Pullman 99164-6410. Approved by the director, College of Agriculture and Home Economics Research Center as Scientific Paper 7927, Project 0617.

The author wishes to thank Dr. Richard $H$. Hart for providing additional references and for contributing valuable comments on the paper.
}

\section{Past Concepts of Carrying Capacity}

In a recent paper, Dhondt (1988) summarized the evolution of the carrying capacity concept and its diverse uses in ecologically based disciplines. After a thorough review of past uses he recommended that the term be dropped from technical use, and used only as a "vague concept." Although the diverse concepts reviewed by Dhondt are confusing, this paper will show that they are all reconcilable within a single generalized concept (and definition) applicable to all systems. In the following analysis, this generalization is achieved working from a sampling of past concepts of carrying capacity.

The Society for Range Management (1989) defined carrying capacity as "the maximum stocking rate possible which is consistent with maintaining or improving vegetation or related resources." Holochek et al. (1989) also stated that carrying capacity or grazing capacity refers to the maximum stocking level possible year after year without inducing damage to vegetation or related resources. Earlier Sharkey (1970) defined it as "the total weight of animals that can be supported permanently." All of the above definitions state or imply an objective. Heady (1975) recommended that definitions such as these be rejected, because they are inapplicable to the multi-product capabilities of rangelands. He stated that as many carrying capacities could be defined as there were management objectives. This idea is a subtle refinement of the concepts of carrying capacity used in early literature of wildlife biology, as summarized by Dasmann (1964) and Macnab (1985), which were still largely based on single implicit, objectives related to population growth curves. However, Heady explicitly differentiated between carrying capacity and grazing capacity, and defined the latter as "the number of animals that produce the greatest return without damage to the physical resources and in concert with other values received from the land." Thus grazing capacity was defined as the carrying capacity required to meet a single, albeit complex objective. Heady went on to state that "optimum carrying capacity expresses the most profitable levels of all products and services, while optimum grazing capacity suggests the most profitable stocking rate."

The analysis by Heady contains essential elements around which to develop a concept of carrying capacity compatible with the multiple objectives and multiple management options of systematic management, but some modifications and simplifications of his approach are needed. There is no need for separate concepts of carrying capacity and grazing capacity, because Heady's concept of grazing capacity fits fully within his definition of carrying capacity. By either name, carrying capacity represents the dependent variable in an objective function - a function subject to many possible objectives and management options. In ecological models, economic models and in general usage carrying capacity is sometimes treated as an independent constraint-an asymptote or a black box, much as any variable may be treated as a parameter for a specific application. But in essence, carrying capacity is an interactive dependent variable because optimization is implied, and its value is dependent on objectives and on other variables.

A carrying capacity represents the rate or level (sensu Forrester 
1961) that should be stocked to achieve specified objectives under specified management options. Carrying capacity is inherently optimized, i.e., it is the optimum rate or level to meet the specified objective(s) under specified management, so the term optimum carrying capacity is redundant. The term carrying capacity suffices and is defined here as the optimum number of individuals or units to achieve specific objectives given specified management options. It is the number of individuals or units associated with achieving an objective or the one which optimally satisfies multiple objectives, given specified requirements and/or options. So carrying capacity is not a maximum number, but an optimized one. If livestock stocking level is optimized to describe a livestock carrying capacity, livestock carrying capacity is then defined as the optimum stocking level to achieve specific objectives given specified management options.

The above conceptualization of carrying capacity may seem so simplified that it has little use. In fact, the definition is a powerful one because it is generalized to meet the needs of all previous ad hoc conceptualizations of carrying capacity; its predecessors are objective-specific cases of the more generalized, optimized concept/ variable described here. In the words of Fuller (1975): "We may hypothesize that information as it increases exponentially-explodes. Conceptuality implodes, becoming even more simplified." The simplified definition of carrying capacity given here has been imploded to provide the power and flexibility to deal with multiple objectives and management options. The optimizations involved in calculating some carrying capacities using current approaches may be complex, but the concept of carrying capacity as an inherently optimized variable has been generalized by simplification.

While the terms optimum carrying capacity, grazing capacity and optimum grazing capacity are thus superfluous, one modifier of the term carrying capacity is justified. Management is based on models, and those models do not always accurately represent the modeled systems. A stocking level may be optimized to a model, but not to the modeled system. So a model carrying capacity calculated through formal optimization (e.g., an optimum stocking level) is an estimate of a real carrying capacity of a modeled system. A system carrying capacity is an idealized concept somewhat like a climax plant community, and calculation of a model carrying capacity, e.g., an optimum stocking level for a management model, is an estimate of the idealized value. For given objectives and options, a model works if its carrying capacity is the same as that for the modeled system, or even if it is closer than that of ill-defined mental models (Forrester 1968).

An ecological system is driven and regulated by environmental and ecological variables which cumulatively reflect the objectives (including human ones, if applied) of the system and, however mysteriously, optimize individual numbers in populations and thereby stocking level. Without human management, objectives are determined by fundamental ecological processes, and choice of management options results from environmental and ecological events and variables. Eventually, individual numbers or stocking level are naturally optimized to a level or cyclical range. Human management imposes new objectives and specific options which can alter system carrying capacity for that species by altering the conditions which determine a system carrying capacity. But while human interaction can affect optimum numbers or stocking levels in a system, humans define the entire optimization when modeling a carrying capacity of a managed or unmanaged system. An understanding of natural objectives of unmanaged ecological systems is needed to model carrying capacities accurately for them.

In modeling carrying capacities of managed systems, human descriptions of environmental and ecological variables and human management options are combined with human objectives, while objectives of natural systems are usually considered as underlying given conditions, even though they are often obscure.

\section{Animal-Units and Stocking Level of Livestock}

Because livestock carrying capacity is often expressed as an optimum stocking level of livestock, and because stocking level of livestock is expressed in either animal-unit-days (AUD) or animalunit-months (AUM) per hectare, a clear concept of an animal-unit is basic to its description. Vague concepts of an animal-unit as a unit of impact or a site-specific concept of it as a unit of intake are incompatible with a multi-objective concept of carrying capacity. The concept of an animal-unit as a unit of energy demand of an animal rather than as a unit describing a plant-animal interaction (Scarnecchia 1985a, Scarnecchia 1986, Scarnecchia and Gaskins 1987) makes possible rigorous quantification of stocking level and carrying capacity.

Stocking level (AUD/ha) is a logical variable to optimize to define a carrying capacity because it describes a cumulative animal demand/land area relationship for a particular land area over some time, but other variables, including number of animals and stocking density (AU/ha) may be optimized. In supply/demand models of range livestock systems, stocking level normally is a level variable (sensu Forrester 1961) while the number of individual animals and stocking density typically are rate variables. Either kind of variable may be optimized to describe a carrying capacity. For objectives involving sustainability, rate variables usually would be optimized. In any case, a carrying capacity number alone is technically meaningless unless the objectives and management details used in optimizing the stocking level or other variable (i.e., calculating the carrying capacity) are specified along with it. Such management details (options) may consist of information on grazing management, classes of animals, breeds of animals, timing of grazing, other stocking variables (Scarnecchia 1985b), silvicultural system, or other simple or complex variables.

Based on the previous discussion, the question "What is the carrying capacity of this land?" is inherently limited in usefulness, because it (1) implies unstated objectives, (2) does not specify chosen management options, and (3) implies a single carrying capacity for a land area. Using the multi-objective concept described here, the question might be rephrased to: "What is the carrying capacity (optimized stocking level, AUD/ha) for ewes and lambs which will meet management objectives $\mathrm{A}, \mathrm{B}$, and $\mathrm{C}$ given management options D, E, and F?" Management objectives could be (1) harvest of $30 \%$ of peak standing crop of all grasses, (2) $10 \%$ defoliation of a specific tree crop, (3) a rate of lamb weight gain/day, and/or (4) any other relevant objective(s). Management options could be (1) a specified grazing system, (2) a specified time of grazing, (3) a specified level of herding, and/or (4) any other management option(s).

The objectives and management options should be at least as specific and detailed as the examples above, and are inherently necessary to estimate meaningful carrying capacities.

\section{Livestock Substitution Ratios}

"How many sheep equal one cow?" Following the same reasoning as in the discussion of carrying capacity, the question might be rephrased more precisely: (1) If X AU of cows will meet single objective A, how many AU of wethers will meet objective A given management option $\mathrm{Z}$ ?; or (2) If Y AU of cows best meet multiple objectives $A, B$, and $C$ under management options $Q$ and $Z$, how many $A U$ of wethers will best meet those objectives using those management options? A livestock substitution ratio may then be defined as the optimum ratio of the number of individual animals or animal-units of one species or class of livestock to the number of animals or animal-units of another species or class to achieve specific objectives, given specifled management options. Generally, a substitution ratio may be defined as the optimum ratio of the 
number of individuals or units of one component to the number of individuals or units of another component to achieve specific objectives, given specified management options. Like a carrying capacity, a substitution ratio is inherently optimized; in "optimum substitution ratio" optimum is superfluous. Also analogous to carrying capacity, model substitution ratios are optimized estimates based on management models, and may or may not be accurate estimates of the substitution ratios of real systems.

Like carrying capacity, a substitution ratio will depend on the management options applied, and will depend on the single variable being equated or the multiple variables being optimized and equated, which are the objectives in its calculation. Just as for carrying capacity, there are many possible objectives; i.e., many different variables can be equated. For example, 4 wethers may have the same total intake (the objective, i.e., the variable equated) as one cow (ratio 4 sheep: $1 \mathrm{cow}$ ) but 10 sheep may be required to harvest as much of a major grass species (the objective) as one cow (ratio 10 sheep: 1 cow). Livestock substitution ratios are not animal-unit equivalents (Scarnecchia 1986), but may be expressed using animal-units. For example, $3 \mathrm{AU}$ of wethers may harvest the same amount of foliage from tree seedlings as $7 \mathrm{AU}$ of cows, under specified management. For that objective and under that management, $3 \mathrm{AU}$ of wethers $=7 \mathrm{AU}$ of cows (ratio $3 \mathrm{AU}$ wethers: $7 \mathrm{AU}$ cows). Some ratios based on single modeled objectives may not be practical to implement because other objectives may not be met. For example, $10 \mathrm{AU}$ of cows may be substituted for $2 \mathrm{AU}$ of wethers to consume an amount of a particular tree seedling, but 10 AU of cows may exceed the carrying capacity for maintaining intake of forage or for some other objective. Specification of objectives and management options is as essential for estimating substitution ratios as it is for estimating carrying capacities.

\section{Applying the Concepts}

The concepts of carrying capacity and substitution ratio described here are compatible with procedures of linear programming and generally with the modeling of plant-animal-environment interactions. They are generalized concepts that may be applied to other products besides livestock. For example, the concepts as defined are capable of describing such variations as human or plant carrying capacities or substitution ratios for plant species. Although flexible, they are rigorous concepts upon which to base increasingly precise management. They are heuristic concepts because they encourage increasingly detailed systems analyses. They can assimilate information as understanding of such systems advances, e.g., the more accurately we can describe the effects of a management option, the more accurate can be our estimates of carrying capacities. The concepts become more useful as we gain more information.
When describing livestock, both concepts are compatible with the animal-unit concept as it should be applied in range livestock systems. If animal-units are used in quantifying either concept, there is greater precision if the AU concept is applied intraspecifically (Scarnecchia 1985a) and describes the energy demand of the animals stocked, because a single number of animal-units cannot contain sufficient information to describe all possible effects on all possible objectives. Further, because an animal-unit equivalent is only one number, specification of intraspecific classes of animals, timing of grazing, and other details will be needed to specify rigorous management options, and estimate carrying capacities or substitution ratios accurately for some objectives.

Scientific management for multiple outputs from range livestock systems or other ecological systems will require other new or redesigned concepts with power to describe multiple objectives and management practices. For rigorous analysis of interactions and synergetic effects, abstractions and boundary limitations will be needed for some concepts. Flexibility, rigor, and power will come from definitional simplicity. If correctly defined, concepts will.be heuristic, and allow better use of data. The concepts of carrying capacity and substitution ratios described here are effectively simplified to accommodate the interactions, multiple objectives, and multiple management options of range livestock and related systems.

\section{Literature Cited}

Society for Range Management. 1989. A glossary of terms used in range management. Denver, Colo.

Dasmann, R.F. 1964. Wildlife biology. John Wiley and Sons, Inc., New York.

Dhondt, A.A. 1989. Carrying capacity: a confusing concept. Acta Oecologia, Oecologica Generalis. 9:337-346.

Fuller, R.B. 1975. Synergetics. Macmillan Publishing Co., Inc., New York. Forrester, J.W. 1961. Industrial dynamics. MIT Press. Cambridge.

Forrester, J.W. 1968. Principles of systems. Wright-Allen Press, Cambridge, Mass.

Heady, H.F. 1975. Rangeland management. McGraw Hill Book Co., New York.

Holechek, J.L., R.D. Pieper, and C.H. Herbel. 1989. Range management. Prentiss-Hall, Inc., Englewood Cliffs, New Jersey.

Macnab, J. 1985. Carrying capacity and related slippery shibboleths. Wildl. Soc. Bull. 13:403-410.

Scarnecchia, D.L. 1985a. The animal-unit and animal-unit equivalent concepts in range science. J. Range Manage. 38:346-349.

Scarnecchia, D.L. 1985b. The relationship of stocking intensity and stocking pressure to other stocking variables. J. Range Manage. 38:558-559.

Scarnecchia, D.L. 1986. Viewpoint: animal-unit-equivalents cannot be meaningfully weighted by indices of dietary overlap. J. Range Manage. 39:471.

Scarnecchia, D.L., and C.T. Gaskins. 1987. Modeling animal-unit-equivalents for beef cattle. Agr. Systems 23:19-26.

Sharkey, M.I. 1970. The carrying capacity of natural and improved land in different climatic zones. Mammalia 34:564-572. 